This is a post-peer-review, pre-copy edited version of an article published in Evidence \& Policy. The definitive publisher-authenticated version is available online at: HYPERLINK "http://www.ingentaconnect.com/content/tpp/ ep/2012/00000008/00000001/art00002" http://www.ingentaconnect.com/ content/tpp/ep/2012/00000008/00000001/art00002

\title{
Perceptions of the Usefulness and Use of Research Conducted in Other Countries
}

\author{
Burchett, H. E. D.*, Lavis, J. N., Mayhew, S. H., Dobrow, M. J. \\ Keywords: "evidence-based decision-making", "evidence use", "evidence \\ types", \\ "semi-structured interviews" \\ ${ }^{*}$ Global Health and Development, Faculty of Public Health and Policy \\ London School of Hygiene \& Tropical Medicine \\ 15-17 Tavistock Place \\ London \\ WC1H 9SH \\ UK
}

\begin{abstract}
Semi-structured interviews in Ghana and England explored perceptions of the usefulness and use of foreign research (i.e. beyond the original study country) compared to locally-conducted research (i.e. conducted in Ghana).
\end{abstract}

There was a preference for locally-conducted studies, although interviewees generally recognised the potential usefulness of foreign research. Various factors affected whether foreign research was considered useful or used; it was 
not used automatically or indiscriminately. Researchers should recognise the potential usefulness of their research beyond the original study country. Further work is needed to explore how to maximise the utility of foreign research, as a means of enabling evidence-informed decision-making where locallyconducted research is not available.

\section{Introduction}

The push for research to inform public health policy and practice is pertinent in low- and middle-income countries, where it is particularly important to ensure that scarce resources are not wasted on ineffective or harmful interventions (Klein, 2000, Chinnock et al., 2005, Santesso and Tugwell, 2006).

In their 1990 report, the Commission on Health Research for Development argued for a distinction between 'global' health research, to generate knowledge and technologies for the control and prevention of ill health, and 'country-specific', 'essential national health research', to address disease profiles and the nature of health problems, health system planning and policy issues (Commission on Health Research for Development, 1990). Global health research (such as that which led to the discovery that mosquitoes transmitted the yellow fever virus) was considered to be based on generally immutable characteristics and so was fairly transferable between countries, whereas 'country-specific' research (e.g. on locally-developed village-based health systems in rural China) was felt to have limited use beyond its original setting. This distinction implied that public health and implementation research were more likely to be 'country-specific', requiring essential national health research to be conducted in each country, whilst biomedical studies would be considered global research and so would be of use to all, regardless of where it was conducted.

Although in an ideal world every country would have the resources to conduct 
all the essential national health research that it needed, in reality this will never be possible. The distinction between global and national health research is useful for the purpose of advocating for more funds and capacity development for 'country-specific' research. However it does little to assist those aiming to encourage evidence-informed decision-making now, since it is never possible to have local research on all the potential topics a decision-maker may be interested in (Frenk and Chen, 2011). Whilst much public health research is context-specific, this does not automatically mean that no lessons can be drawn from research conducted in other countries. A deeper examination of perceptions of the utility of research conducted elsewhere (i.e. foreign research) is therefore needed. As such, a study conducted on Nigeria will be 'foreign research' for Ghanaian decision-makers, whilst a study conducted on Ghana being considered by Ghanaian decision-makers will be referred to as 'locallyconducted research'. The current study explored perceptions of the usefulness and use of foreign research (as opposed to locally-conducted research). We did not attempt to examine other related issues, such as the nationality of researchers working in Ghana, or where they were based.

Little is known about whether foreign public health or policy research is considered to be useful or used by public health decision-makers (although a study of the usefulness of clinical research found that clinicians valued locallyconducted research more highly than foreign research) (Guindon et al., 2010). Enhanced understandings of these perceptions of usefulness can help to maximise the 'added value' of research, by helping to encourage the use of studies beyond their original setting. It could also help to encourage the appropriate use of foreign research, enhancing the potential evidence base which decision-makers could use.

The concepts of 'usefulness' and 'use' are both subjective terms (Weiss and Bucuvalas, 1980). This paper assumes that the perceived usefulness of research is one of the factors that may affect whether research is used. Research can be 'used' in a number of ways - either instrumentally (to solve a 
particular problem at hand), conceptually (to think through how to approach a problem) or symbolically (to justify a choice made for other reasons) (Weiss, 1979). However it is difficult to objectively identify and measure every episode of research 'use'. Therefore this paper aims to explore perceptions of the usefulness and use of research, as factors that may affect actual research use. This article is based on data from a larger study which aimed to explore perceptions of the usefulness and use of research for public health decisionmaking in Ghana. The specific objectives for this part of the study were to:

Identify how decision-makers find out about research Understand the value placed on research, or the perceived importance of research use in decision-making Explore the extent to which research is perceived to be used Identify the types of research considered useful, or used, in decision-making Explore the usefulness of existing 'international' conceptualisations of research (e.g. 'global' versus 'country-specific' and research use in decision-making

Assess whether such perceptions vary depending on the type of researcher or decision-maker

This article focuses on perceptions of the usefulness and use of foreign research in Ghana, as well as the perceived usefulness of Ghanaian research in other countries (perceptions of the usefulness and use of locally-conducted (i.e. Ghanaian) research are presented elsewhere).

\section{Methods}

Semi-structured interviews were conducted with both decision-makers (from Ghanaian national and sub-national government organisations and agencies and other stakeholders working in the field of public health in Ghana) and researchers (from Ghanaian and British institutions). Interviewees were 
identified through purposive sampling.

All the interviews were conducted by the first author and lasted 20-80 minutes (though most were 45-60 minutes long). All interviewees were interviewed once only; in two cases the interviewee chose to involve a colleague in their interview (i.e. two interviewees participated in the one interview).

Interviewees were asked about their perceptions of the usefulness and use of research, which led onto more specific questions about foreign research (e.g. 'thinking as well about research that's been conducted in other countries outside of Ghana, do you often come across that research and if you do, how useful do you find that for your work?'). Those who conducted research were asked whether they believe their research had been used, or who they thought might find it useful, which also led on to specific questions about whether it could be of use elsewhere (e.g. 'thinking back to your research, do you think it could be useful to people in other countries?'). The interviewer did not define 'research', 'usefulness' or 'use', but rather looked to the interviewees to define the terms themselves.

The interviews were digitally recorded except in two cases when permission was declined by the interviewee and a third where the equipment failed during the interview. In these cases, notes were taken during the interview and typed up in more detail immediately afterwards. Recorded interviews were transcribed verbatim.

The transcripts were analysed using Framework Analysis and 'Atlas.ti' software was used to manage the data (Ritchie and Spencer, 1994). A coding framework was developed through an initial analysis of five interview transcripts, to identify themes emerging from the data. These were then discussed with the third author and cross-checked with the study's aim and objectives to ensure that they were all covered by the identified codes; additional codes were added as necessary. The first author then coded all of the transcripts. After applying the coding framework to all the transcripts, the coding framework was revisited. 
Some codes were merged, if too similar, whilst others were recoded with subcodes if they were too broad. The coded transcripts were then summarised into excel spreadsheets (whilst attempting to use the same language and terminology as the interviewee), where one column was allocated for each code and each row represented an interviewee. Each column (code) was then sorted according to the type of interviewee and explored for patterns within the data and between types of interviewees or responses. The original transcripts were constantly referred back to throughout the process, to ensure that the study findings were grounded in the data and coded text was not taken out of context.

The London School of Hygiene and Tropical Medicine and the Ghana Health Service's Health Research Unit both gave ethical approval for the study. Interviewees were given an information sheet describing the study and were asked to sign a consent form before commencing the interview.

\section{Findings}

Sixty-seven interviews were conducted with 69 interviewees between February 2008 and March 2009 in Ghana and England. The study sample comprised 13 national-level government staff, 12 sub-national government staff, 18 stakeholders (e.g. development partner and non-governmental organisation (NGO) staff), 24 researchers (two of whom directed international research institutions which had conducted studies in Ghana) and two others, who had conducted research and also held government positions (either currently or previously). Most interviewees were Ghanaian, but some came from Europe $(n=6)$, United States of America $(n=2)$ and other African countries $(n=2)$.

The findings will first summarise interviewees' definitions of research, before exploring their awareness of foreign research. Perceptions of the usefulness and use of foreign research will then be discussed, before consideration of the routes and reasons foreign research may be used in Ghana. The use of Ghanaian research in other countries will then be discussed, before 
considering the potential for Ghanaian research to be considered 'foreign' if conducted in a different setting within Ghana. The types of foreign research that were perceived to be useful and used will then be discussed, before summarising the conditions that were felt to affect whether foreign research would be considered useful or used.

Definitions of research were frequently wide, including formal studies as well as routine data, government reports or any informal investigation (e.g.

observations, talking to people). However the latter tended to be conducted by the interviewee within Ghana and so rarely appeared to be incorporated in concepts of 'foreign research'.

\section{Awareness of foreign research}

When asked about their awareness of research in general, most interviewees focused on their awareness of locally-conducted research, to the neglect of research conducted elsewhere. Some government staff mentioned international conferences as one means of hearing about research (which would include foreign research), though one pointed out that "you are not always going on international conferences" [007, government staff]. Non-Ghanaian stakeholders seemed to be more aware of foreign research findings because they subscribed to listserves and received updates from their international headquarters. Some less senior subnational government staff lacked internet access, which may have limited their access to foreign research.

\section{Usefulness and use of foreign research}

When asked general questions about the usefulness and use of research, interviewees tended to focus on locally-conducted research; only on probing 
specifically about research from other countries did they discuss these issues in relation to foreign research. Although there was frequently either an implicit or explicit preference for locally-conducted research, many interviewees felt that foreign research was useful for, or used in, Ghanaian policy-making. Some interviewees gave examples of national policies that were directly informed by evidence from other countries. For example, a few interviewees noted that Ghana's policies aiming to increase rates of skilled attendance at birth (by a specialist health professional) were based on evidence from other countries.

The usefulness and use of foreign research was viewed negatively by certain interviewees. This was either because the interviewees were not convinced by the research itself, or because they did not feel that it was applicable/ transferable to the Ghanaian context but rather that it had been 'forced' upon them. For example, the quote below describes an interviewee's disagreement with Ghana's adoption of the ('evidence-based') international policy of excluding traditional birth attendants (TBA's) from definitions of skilled attendants and, more generally, their exclusion from policies to reduce maternal mortality.

“...because the international community says no, so in Ghana, we say no. You know, which means the international community does not recognise the reality we live with here..."

047, academic researcher

However not all interviewees felt that foreign research was useful or used. Some pointed out that learning from other countries was not limited to research, for example through exchange visits or simply learning from others' experiences. Some felt that exchange visits would be more influential than 
research from elsewhere. These visits tended to look at policy options and implementation issues.

Routes to, and reasons for, foreign research use

Some felt that foreign research may be used more than locally-conducted studies, either because of a lack of Ghanaian research production or because of the strong influence of external agencies.

It was recognised that foreign research may be influential in Ghana because it was used to inform international policies which were adopted incountry (i.e. rather than the research influencing Ghanaian policy directly).

A few indicated that decision-makers would be unlikely to use foreign research, explaining that they would only use it if the research was introduced through international organisations (this was even felt to be true for studies conducted in Ghana). A minority suggested that research findings were more likely to be accepted or adopted if they were introduced through international organisations.

“I don't think policy-makers will go and read about other countries. So it's, international organisations like WHO, UNICEF and all the others who would bring all these other findings which may be relevant for various countries to a level where different governments can pay attention to them."

015, researcher

\section{Use of Ghanaian research elsewhere}

Interviewees recognised that the issues around foreign research were not unidirectional; the usefulness and use of Ghanaian research in other countries, as well as at the global level, was noted by many. 
"the kind of research we do, actually has an international nature. So findings from here can be translated to settings similar to our own here"

038, researcher

A few interviewees expressed the opinion that not all research conducted in Ghana was even of use to Ghanaians themselves, but nevertheless may be of use elsewhere. This was because research agendas were set by funders or researchers external to Ghana, who were not aware of factors limiting the usefulness of study findings (e.g. if legislation prohibited it).

\section{Foreign ‘Ghanaian’ Research}

Questioning the utility of research carried out in one setting to another was also not limited to the country-level: interviewees noted issues around the usefulness and use of research conducted in one Ghanaian setting to other settings in the same country. Those producing sub-national research in Ghana often felt it could be of use to other districts or regions in Ghana. However several interviewees noted that there were differences within Ghana that meant that research conducted in one setting may not necessarily be of use in another, for example due to differences between urban and rural settings or cultural differences between populations in different areas.

\section{Types of foreign research perceived useful/used}

Certain types of foreign research were more likely to be considered useful or used, notably efficacy studies or evaluations of 'simple' interventions. When discussing foreign research use, the examples that interviewees used were often biomedical or clinical studies, rather than public health research. This suggests that biomedical/clinical research was more likely to be considered of 
use in settings 'foreign' to the original research.

Some interviewees felt that multi-country studies were a more useful form of foreign research, arguing that they may "roll out quicker" than studies conducted in a single foreign country.

Foreign studies that helped to inform decisions around policy options were considered more likely to be useful/used than those which described or measured health problems (e.g. prevalence studies) or addressed implementation issues.

Even amongst those who believed that foreign research was of use, the need for locally-conducted research was still recognised, particularly during implementation.

“...we were going to write a policy on malaria and the drugs. There were a lot of African countries that had already started using the combination drugs...it wasn't very, very necessary to do a wide scale research to find out because its, the evidence is there....Some of the researches have already been done [outside of Ghana], it's been proven. There's no point going to also repeat the whole thing. But whilst you are implementing you can still, you know, do some operations research to make sure that it's [implemented well]"

030, government staff

\section{Conditions affecting the use of foreign research}

Foreign research was not felt to have been used indiscriminately, nor was it automatically considered useful. Some interviewees pointed out that foreign research would be used if no research had been conducted on a particular topic within Ghana. Others explained that whether or not foreign research was 
useful or used depended upon the topic, as well as where it had been conducted. Some explained that as long as the situations were similar, lessons could be drawn from foreign research.

Assessing the similarity of the Ghanaian context to the original research study setting, or determining whether or how to adapt a study, were mentioned as necessary steps when deciding whether or not foreign studies may be of use.

"But clearly I think research done in other countries, especially countries that have similar characteristics as Ghana, I mean, are quite useful or studies that are multi-country studies, quite useful."

017, stakeholder

A few mentioned that regardless of the strength of evidence from elsewhere, any public health policy or intervention should still be evaluated within Ghana, either before or upon introduction.

\section{Discussion}

When discussing the usefulness and use of research in general, there was a tendency for interviewees to focus on locally-conducted research, neglecting to consider foreign studies without additional probing by the interviewer. This suggests a preference for locally-conducted research over foreign research. The inclination towards locally-conducted research has also been found in other studies. Adjei et al.'s study on the information needs of policy-makers in Ghana focused more on Ghanaian research than foreign (Adjei et al., 2001). Woelk et al.'s study in three other African countries also found a preference for local research, with studies conducted elsewhere perceived as "distant" [p10] (Woelk et al., 2009). Studies of clinicians from low- and middle-income countries reported that they felt that they were more likely to change their practice based on locally-conducted or published research, rather than that 
from elsewhere (Page et al., 2003, Guindon et al., 2010). The fact that clinicians reported this is an interesting point to note, since the findings presented in the current study suggest that clinical research was more likely to be considered useful beyond its original setting than public health research.

That several interviewees discussed biomedical or clinical studies (rather than public health research) when asked about the usefulness and use of foreign research implies that this type of research may be more likely to be considered of use beyond the original setting in which it was conducted. Biomedical and clinical research, with relatively short causal pathways and based on the greater likelihood of universal biological mechanisms, is generally considered easier to apply and transfer from one setting to another. In contrast, public health interventions are thought to rely on more context-sensitive behavioural and interpersonal mechanisms and organisational or structural characteristics. Whether foreign research was perceived to be useful or used was affected by the existence of locally-conducted research, the influence of external agencies, the research topic and perceived similarities with the research country. Despite the preference for locally-conducted research, when probed it became clear that foreign research was considered useful and was used in Ghana, although neither its appropriateness nor its use were considered automatic. There were strong views that it should not be used indiscriminately, pointing to the importance of understanding how to assess whether research from one setting could be of use elsewhere.

Those who felt that all complex public health interventions would need to be evaluated in-country, regardless of the existence of foreign research, clearly conceptualised there to be a need for locally-conducted research. However not all held such simple, dichotomised views; many recognised that public health research could be of use beyond its original setting.

There appeared to be an understanding of the need for both global and locallyconducted public health research, since there was recognition that foreign 
research may be useful in Ghana, albeit particularly when complemented by locally-conducted research. An appreciation of the importance of locallyconducted research for decision-making has previously been presented in the academic literature (Commission on Health Research for Development, 1990, Lomas et al., 2005, Lewin et al., 2009, Dobrow et al., 2006, Behague et al., 2009). However the international research and policy communities do not appear to have fully accepted this, given the lack of specific funding available for locally-conducted research whose agenda has been set by those within the country.

Efforts to develop national health research systems focus on the generation of research nationally, to the neglect of strategies to enhance capacity to identify and consider the usefulness of foreign research (Pang et al., 2003, Kirigia and Wambebe, 2006). Whilst there is clearly a need to increase the production of locally-conducted research, there also remains a need to recognise the potential utility of foreign research. Although there has been recent recognition of the need for this capacity, our understanding of how to assess whether research from elsewhere could be of use, or how to successfully adapt evidence to new settings, is lacking (Frenk and Chen, 2011, Burchett et al., 2011). Developing greater understandings of the factors that may influence the perceived usefulness of foreign research could help to enhance the production and reporting of studies so as to maximise their potential usefulness elsewhere. Tools have been developed to help assist decision-makers in judging whether foreign research may be of use in their setting, however these are relatively new and untested as yet (Burchett et al., 2011). Improved understandings of perceptions of usefulness could help to refine these tools which, combined with building capacity for assessing when foreign research may be of use, should help to encourage the appropriate use of such research amongst a wider audience, so enhancing evidence-informed public health.

This study focused on one country and so the extent to which the findings may be reproduced in other countries is uncertain. However the study included a 
large sample, with a wide range of actors involved in research and policy in Ghana. It is hoped that the detailed exploration presented here can be used to further develop and test these issues in other contexts. This will help to improve existing tools for assessing foreign research's utility, as well as to develop strategies to help researchers maximise the utility of their studies and to strengthen decision-makers' capacity to conduct such assessments.

\section{Conclusions}

Despite a preference for locally-conducted research, there was general acceptance that foreign research could be useful and was used in Ghana, although this was not automatic. Better comprehension of the factors influencing perceptions of the usefulness and use of research from other countries could help to enhance the perceived usefulness of research beyond its original study setting. This will strengthen the role of research in improving health across the globe.

\section{References}

Adjei, S., Cofie, P. K., Addo, D. B., Essegbey, E. T., Vordzorbgbey, E. \& Larbi, E. 2001. "How Can We Act on Information We Don't Know": A study into information and communication needs and use of research information in health policy decisions in Ghana.

Behague, D., Tawiah, C., Rosato, M., Some, T. \& Morrison, J. 2009. EvidenceBased Policy-Making: The implications of globally-applicable research for context-specific problem-solving in developing countries. Social Science and Medicine, 69, 1539 - 1546.

Burchett, H. E. D., Umoquit, M. J. \& Dobrow, M. J. 2011. How Do We Know When Research from One Setting Can Be Useful in Another? A Systematic Scoping Review of External Validity, Applicability and Transferability Frameworks. Journal of Health Services Research and 
Policy, in press.

Chinnock, P., Siegfried, N. \& Clarke, M. 2005. Is Evidence-Based Medicine Relevant to the Developing World? PLoS Medicine, 2, e107.

Commission on Health Research for Development 1990. Health Research:

Essential link to equity in development. Oxford: Oxford University Press.

Dobrow, M. J., Goel, V., Lemieux-Charles, L. \& Black, N. A. 2006. The Impact of Context on Evidence Utilization: A framework for expert groups developing health policy recommendations. Social Science and Medicine, 63, 1811 - 1824.

Frenk, J. \& Chen, L. 2011. Overcoming Gaps to Advance Global Health Equity: A symposium on new directions for research. Health Research Policy and Systems, 9, 11.

Guindon, G. E., Lavis, J. N., Becerra-Posada, F., Malek-Afzali, H., Shi, G., Yesudian, A. K., Hoffman, S. J. \& For the Research to Policy and Practice Study Team 2010. Bridging the Gaps Between Research, Policy and Practice in Low- and Middle-Income Countries: A survey of health care providers. Canadian Medical Association Journal, 182, 362 - 372.

Kirigia, J. M. \& Wambebe, C. 2006. Status of National Health Research Systems in Ten Countries of the WHO African Region. BMC Health Services Research, 6, 135.

Klein, R. 2000. From evidence-based medicine to evidence-based policy? Journal of Health Services Research and Policy, 5, 65-6.

Lewin, S., Oxman, A. D., Lavis, J. N., Fretheim, A., Marti, S. G. \& MunabiBabigumira, S. 2009. SUPPORT Tools for evidence-informed policymaking in health 11: Finding and using evidence about local conditions. Health Research Policy and Systems, 7, S11.

Lomas, J., Culyer, T., Mccutcheon, C., Mcauley, L. \& Law, S. 2005. Conceptualizing and Combining Evidence for Health System Guidance. Ottowa: Canadian Health Services Research Foundation.

Page, J., Heller, R. F., Kinlay, S., Lim, L. L., Qian, W., Suping, Z., Kongpatanakul, S., Akhtar, M., Khedr, S. \& Macharia, W. 2003. Attitudes of developing world physicians to where medical research is performed and reported. 
BMC Public Health, 3, 6.

Pang, T., Sadana, R., Hanney, S., Bhutta, Z. A., Hyder, A. A. \& Simon, J. 2003. Knowledge for Better Health - A Conceptual Framework and Foundation for Health Research Systems. Bulletin of the World Health Organisation, $81,815-820$.

Ritchie, J. \& Spencer, L. 1994. Qualitative Data Analysis for Applied Policy Research. In: BRYMAN, A. \& BURGESS, R. G. (eds.) Analyzing Qualitative Data. London: Routledge.

Santesso, N. \& Tugwell, P. 2006. Knowledge Translation in Developing Countries. Journal of Continuing Education in the Health Professions, 26, 87-96.

Weiss, C. 1979. The Many Meanings of Research Utilization. Public Administration Review, 39, 426 - 431.

Weiss, C. H. \& Bucuvalas, M. J. 1980. Social Science Research and DecisionMaking, New York, Columbia University Press.

Woelk, G., Daniels, K., Cliff, J., Lewin, S., Sevene, E., Fernandes, B., Mariano, A., Matinhure, S., Oxman, A. D., Lavis, J. N. \& Lundborg, C. S. 2009. Translating Research into Policy: Lessons learned from eclampsia treatment and malaria control in three southern African countries. Health Research Policy and Systems, 7, 31. 
